\title{
A MODULAR SIMULATION MODEL FOR ASSESSING INTERVENTIONS FOR ABDOMINAL AORTIC ANEURYSMS
}

\author{
Christoph Urach \\ Günther Zauner \\ dwh simulation services \\ Vienna, AUSTRIA
}

\author{
Gottfried Endel \\ Ingrid Wilbacher \\ Main Association of Austriann \\ Social Insurance Institutions \\ Vienna, AUSTRIA
}

\author{
Felix Breitenecker \\ Institute of Analysis and Scientific Computing \\ Vienna University of Technology \\ Vienna, AUSTRIA
}

\begin{abstract}
This paper discusses the development of an individual based simulation model for evaluation of interventions for better treatment of patients with abdominal aortic aneurysms (AAA). The interdisciplinary subject required collaboration of medical doctors, Health Technology Assessment (HTA) experts and modelers. The here presented modular model structure is flexible enough to allow adaptation on screening research questions for similar diseases. Another focus of the work was integration of risk factors and how it determines our model choice, especially because steadily increasing knowledge about or improved treatment of AAA could cause necessity of reevaluation. Through inclusion of several patient specific properties the model does not only provide comparison of current state with screening but also elaboration of alterations of population characteristics and its consequences on AAA cases.
\end{abstract}

\section{INTRODUCTION}

Austrian reimbursement data shows that most of aortic aneurysms concern the infrarenal aorta. Incidence is highly increased amongst men (about 5:1 (Iribarren et al. 2007)) and generally increasing with age. Over $85 \%$ of abdominal aortic aneurysms, furthermore denoted AAAs, occur within people older than 65 years therefore changing population structure and increasing life expectancy leads the focus of attention of national health institutions on treatment of AAAs.

One major problem of AAAs is their usually asymptomatic course of disease. When the aorta ruptures most of the patients die on their way to hospital (Group 2002) therefore the question arises which influence diagnostic interventions have on AAA care and costs from a payers perspective. For this goal a model was developed to

- simulate development, treatment and interventions like screening strategies (see figure 1),

- analyse incidence of AAA cases and corresponding costs for 65-year old people over the next 20 years,

- consider the influence of key factors for AAA growth and rupture risk and

- compare continuation of current practice with organized screening strategies. 
Screening for AAAs was also investigated by EUnetHTA (European network for Health Technology Assessment). Classification of AAAs and the main screening strategy are inherited from its work. Similar studies, e.g. (Kim et al. 2007), used Markov models for the health economic analysis of organised AAA screening, whereas we present a flexible Agent Based Model which proved to be better suitable for our purpose of regarding additional properties of patients (see section 2) as well as the possibility to provide a modular framework (section 3) which is easily adaptable for answering screening questions for other, from a modellers perspective similar, diseases. Parametrization was mainly addressed in the IFEDH-project (FFG grant number 827347) within an interdisciplinary group consisting of modellers, medical doctors and HTA experts through systematic literature research, expert opinions and reimbursement data analysis. Results of this project are subsequently frequently cited.

The remainder of this paper includes results of the base simulation run and a limitations of the model followed with conclusions in section 6 .

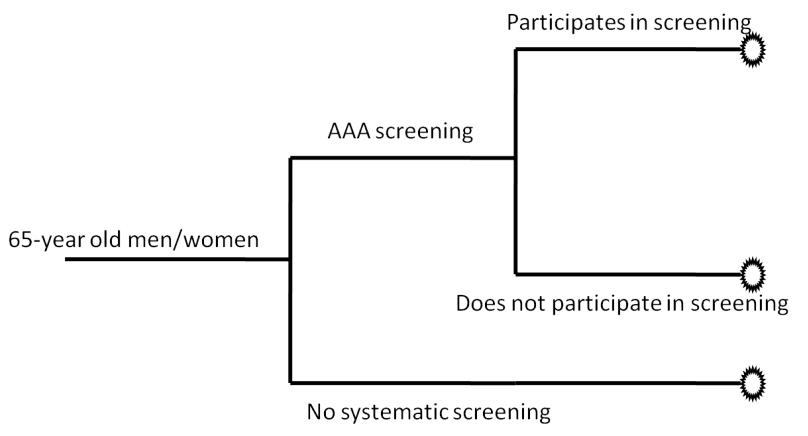

Figure 1: Evaluated intervention: No systematic screening vs organized screening

\section{MODEL CHOICE}

Abdominal Aortic Aneurism is a disease which can be developed by anyone although its development is favored by several factors. Disease progression, represented by a person's health state, is usually dependent on diameter of the abdominal aorta. Detection is usually done by ultrasonic examination. The model serves various purposes. Looking at the disease, detection and health economic questions (see section 1) the model should be able to simulate

- the target population for abdominal aortic screening programs.

- the development of aortic growth and / or corresponding health state

- the influence of identified risk factors on disease progression

- effects of treatment (surgery)

With the information generated by a model satisfying these requirements it is possible to estimate the future effects of screening on AAA related deaths and corresponding life years gained. Furthermore it is possible to analyze the effects of other interventions targeting risk factors (e.g. smoking cessation programs). If costs are included also cost-effectiveness analysis can be performed.

Given the defined problem the best modeling method to efficiently, transparently and flexibly get results has to be chosen. Efficiently because the amount of time and effort needed to create and implement such a model must not be in relation to expected information gain. Transparently because it must be possible to explain to third parties what the model actually doeswithout forsaking accuracy. Flexibility is mandatory for expanding the model in case of more knowledge and therefore expended assumptions, for example inclusion of further risk factors. 


\section{Urach, Zauner, Endel, Wilbacher, and Breitenecker}

Regarding the HTA questions two modeling methods were considered: Markov Models and Agent Based Models (as basis for the microsimulation). The former can be realized faster whereas Agent Based Models provide much more flexibility. Considerations about the functional requirements of the model determine which method suits our purpose better.

The target population for the evaluation of screening programs is minimal a strictly defined cohort which is distributed over a predefined state-space with certain probabilities. For a small amount of possible states as well as homogeneous treatment of people this perception is very transparent. Using decision trees for visualization the choices which lead to the propagation from one to another state and the fast computation time favor the use of Markov Models for this specific requirement. For some purposes like simulating both men and women within one model we need to distinguish between groups of people depending on several factors. Looking back at the cohort simulation approach, each additional property assigned to people leads to the introduction of a multiple of already used states. Each time the whole model structure has to be reworked, restricting flexibility to introduce further factors. With the introduction of too many different states also the originally transparency gets absurdly confusing and complicated. Agent based approaches on the other hand can look at individuals. If all people are treated the same way and share the same properties it seems unjustified to put in effort to build a population model which creates individuals which together are the target population. As soon as other factors are included this approach starts to shine: The factor is introduced as agent property, every agent can be in its own state without changing the overall model structure at all. Already knowing that at least sex and age are needed in the model with the possibility of requiring even more risk factors (Sweeting et al. 2012) to properly assess development or abdominal aortic aneurysms in Austria's population this method suits our needs far better.

Summarizing all considerations about modeling method choice regarding needed structure on the one hand and flexibility on the other hand resulted in the decision to use an Agent Based Model whereas each agent is interpreted as an individual person. Choosing the technical properties of agents and their environment carefully allows the flexibility to easily define further scenarios or add other risk factors without the need to touch the model structure. It is not the purpose of the model to simulate development of incidence and prevalence in the overall population, but only the specific screening age class, although using the developed model this is easily possible by expansion of the agent properties of the population module.

\section{MODEL STRUCTURE}

Having decided to use an individual based approach the structure for implementation regarding previous deliberations has to be developed. The model is divided into four sub-models (see figure 2) furthermore called modules, referring to four thematically different fields, which interact with each other:

- Population module

- Disease progression module

- Treatment module

- Protocol module

The population module initializes the targeted population and simulates its progression over the modeled time horizon (in the base case 20 years), for example aging or non-AAA-related death. Disease progression, in this case the growth of the abdominal aorta, is implemented in the eponymous module. Surgeries belong to the treatment module while the protocol module is responsible for the recording of values of interest.

The concept behind this structure lies in flexibility and re-usability. Wanting to simulate the same model for another country just needs exchange or adaptation of the population module. Disease or treatment specific assumptions only need to be changed within the corresponding modules. It is even possible to simulate another disease by exchanging just these two modules. The protocol serves two purposes. It records values and performs analysis, for example the calculation of costs of life years gained. After this 
short overview the modules are now explained in detail.

\section{Protocol and Evaluation}

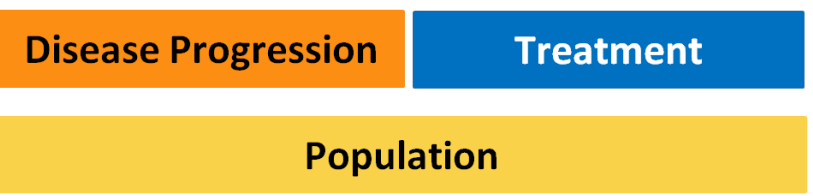

Figure 2: Modular Structure of the Model

\subsection{Population Module}

For the purpose of evaluating a screening program for a specific cohort (for example all 65-year old male people) it would suffice just creating the corresponding number of agents and defining their non-disease specific mortality rate over the course of time. However, we want a much more general module where the target population can be changed on demand. If we first look at the 65 -year-old population and evaluate a specific screening program it should be further possible to investigate certain sub-groups, for example 65-year-old male smokers, by just setting the parameters responsible for the choice of the investigated people. Therefore it is necessary to model the whole population (in this case of Austria) together with the properties for selecting sub-groups.

The population modules executes several tasks (3):

- It initializes the actual population: A predefined number of agents which can be regarded as individuals are created. Sex, age and smoking status are assigned to the agents to resemble the corresponding distribution for the target country, in this case Austria. Providing distribution of further factors (which can be dependent on existing factors) allows adding arbitrary more.

- Life expectancy tables, data on migration, birth tables and assumptions on changes of risk factor distributions provide the information to simulate the future development of the population.

- Through the course of the simulation agents are born, they get older and can die. They are also able to develop risk factors. In this case they can start to smoke, change their smoking habits, resulting in a change of addiction severity, or stop smoking to create their smoking history.

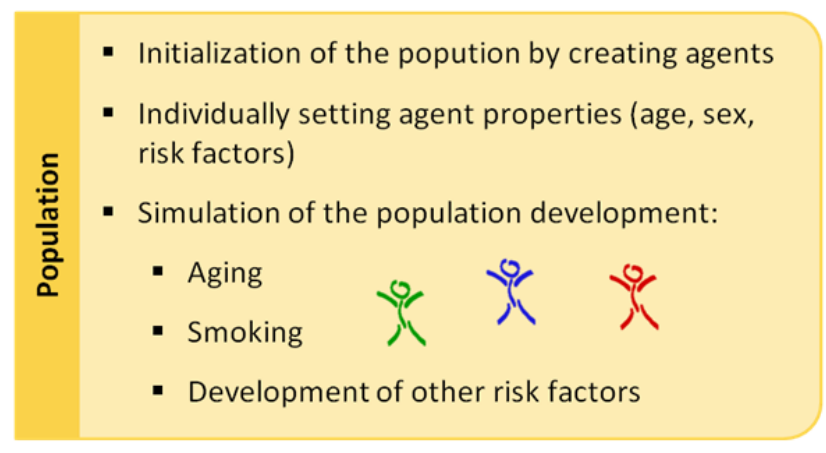

Figure 3: Tasks of the population module 


\subsection{Disease Progression Module}

One module has to reflect disease progression which is in the case of the chosen approach individual for each simulated agent. In the case of AAA screening it manages the aorta diameter and events like rupture. The growth is calculated with a function of actual size, patient characteristics and risk factors. Risk of rupture increases with the abdominal aorta diameter and can be classified as Small/Medium/Large AAA corresponding to Small/Medium/Large risk of rupture. This aggregation to three (or four when including no AAA) states is not model immanent but used for presentation and comparison purposes as it is the usually used classification of AAA disease severity (Kim et al. 2007).

\subsection{Treatment module}

The treatment module manages emergency or elective treatments and also other possible longer-term interventions, like smoking cessation programs or screening. As for medical treatment in hospitals there only exists surgery. Elective surgery is usually performed when the aneurysm exceeds a certain $(5.5 \mathrm{~cm})$ size and the patient is fit for surgery. Depending on the aneurysm location, its appearance as well as patient characteristics like health state and age, either endovascular or open surgery is chosen. This choice also influences future treatment course.

When an AAA ruptures, the patient undergoes, when reaching the hospital alive and viable, emergency surgery. In this module the assumption, based on evaluations of reimbursement data, is used that short-term mortality for these patients is far higher than that of corresponding patients which have an elective surgery, but if the former survive the operation and initial hospital stay the long-term development is the same.

From a technical point of view screening can be seen as special type of treatment because it also can alter natural disease progression (not screening itself, but the behaviour of agents diagnosed with AAA).

\subsection{Protocol Module}

Previously described model parts together are sufficient to simulate dissemination of AAA within a defined population as well as the impact of treatment and interventions. Analysis of results requires a program part which documents every event of interest over the whole simulation time horizon. Results of the model are on a patient level, meaning that for every individual at each time-step every realization of simulated health states is recorded (smoking status, AAA diameter size, treatments, whether its AAA status is known incidentally, from organized screening or not known, rupture, age, health state, AAA specific costs).

As the model results shall provide insight on the effect of introducing organized screening for 65-year old people using the follow-up screening strategy proposed by EUnetHTA compared to the current state outcome measures for evaluating the benefit have to be defined. In this case life-years gained as benefit for the patients as well as costs per life-years gained (quality adjusted life-years gained will not be used as usually there is no reduced quality of life before rupture) for cost-effectiveness analysis are used.

The model output, numerous spreadsheets, is used to create key figures. Our main results for evaluation of the effectiveness of organized screening introduction versus current practice are prevented ruptures, prevented death cases and the incremental cost-effectiveness ratio (ICER) as additional costs for the national health insurance system per saved life year. We also evaluate other values, for example the number of people occupying each health state, which are used for both model validation as well as to create additional knowledge about the impact of interventions. The protocol module processes this task. Costs and the utility function, which consists in this case of accumulated life years, are appointed for all states or events of interest within the model for later evaluation and comparison. 


\subsection{Model Progression}

Development of the model concept and structure for AAA-screening requires detailed knowledge on possible succession of events. Modeling technique specifies how this model progression is realized, whether using artificial time or otherwise triggered updates. Substantiating our choice we consider

- Modeling Method

- Model Structure

- Data Knowledge

which we already agreed on and are therefore predetermined. They are strongly interdependent and strongly influence our deliberations. Updates of the states can happen continually, after time-steps or even be mixed, for every section or state differently. Considering intelligibility as well as analytical investigation updating the system after continuous or fixed, equally long, time steps both seem plausible. For screening intervals as well as protocol intervals discrete time-steps are obligatory. The basic model structure consists of agents representing patients with various attributes which are frequently updated. Time points of interest are whenever the state of the whole system should be observed or when events cause change of attributes therefore the time-steps must not be too big. Choosing very small time-steps eludes this problem but causes several others: First the required computational power increases. Second identification of the parameters available in a much coarser way have to be converted or interpolated which could lead to complicated formulas their solutions being hardly interpretable in the real world. As already addressed this problem often goes hand in hand with available data and knowledge on the system structure.

Regarding AAA-screening the most important attribute is the size of the aorta of a patient. Although its growth is continuous it is measured by sonographic investigation. Some studies (e.g. (Kim et al. 2007)) suggest screening of the most endangered patients every three to six month whereas other patients belonging to the risk population, with an AAA-diameter bigger than $30 \mathrm{~mm}$, are screened far less. Every identified screening interval which seems plausible is divisible by three. Regarding a medical checkup and screening programs three months therefore is the biggest possible time-step without losing or abandoning further information.

We still have to consider other influencing factors, in this case other risk factors like smoking or chronic diseases and determine whether the chosen time-intervals are granular enough to describe their development sufficiently for example additional risk factors like changing smoking habits influence AAAgrowth significant encouraging the use of continuous time. Results for economic evaluations on the other hand have to be accumulated over a period of time for example year-wise or, when comparing scenarios, for patients participating at the screening program from its start till they die, so when using smaller time-intervals results have to be aggregated.

Recapitulating gathered information, assumptions, model structure and technique led to usage of a hybrid approach. At model start the population is initialized. Each person has its own set of states (e.g. age, sex, smoking status) and an aorta diameter corresponding to them. After model start aorta diameter grows according to a function depending on the patient parameters and history. Depending on the patients attitude towards screening they participate at the organized screening program, alternatively an AAA can be detected by chance. If a persons AAA is detected it may alter habits (e.g. smoking) which results in a different aorta growth function. However, people with detected large AAAs undergo, if eligible, surgery, which are triggered by discrete events. The aorta of all patients with an AAA and no surgery can rupture which results in emergency operation or death. The protocol module documents a snapshot of all states and occurred events every 3-month.

\section{PARAMETRIZATION}

Parameterization of the model is mainly performed based on information created within the IFEDH project (FFG grant number 827347) whereas details can be looked up in the corresponding reports as well as 
EUnetHTA core information (EUnetHTA 2012). This information was supplemented with reimbursement data as well as results from studies. Aggregation of the collected data to technical parameters is also described within these reports. Assumptions for screening strategies originate from both EUnetHTA core information as well as the Main Association of Austrian Social Security Institutions. Concerning costs we use a payers perspective.

\section{IMPLEMENTATION}

The model is implemented in Anylogic which uses an object oriented approach to realize above explained modules. The Main class 4 manages and regulates the settings for the program, for example the number of agents and their starting configuration which is committed to the population module. Aorta growth is realized solving a differential equation in the disease module within each agent. Other parameters, which can also influencing the growth rate, are scheduled by discrete events thus leading to a hybrid model. One observed problem with this approach was the drastic slowdown (about 1:10) of the whole model as soon as aorta diameter growth is modeled in this continuous way. As it still runs fast enough (about 1 minute on a Xeon E31245 CPU paired with 8GB RAM) with 89982 agents (which represents the sub-population of interest in the Austrian screening setting) we accepted this drawback in favor of more accurate results.

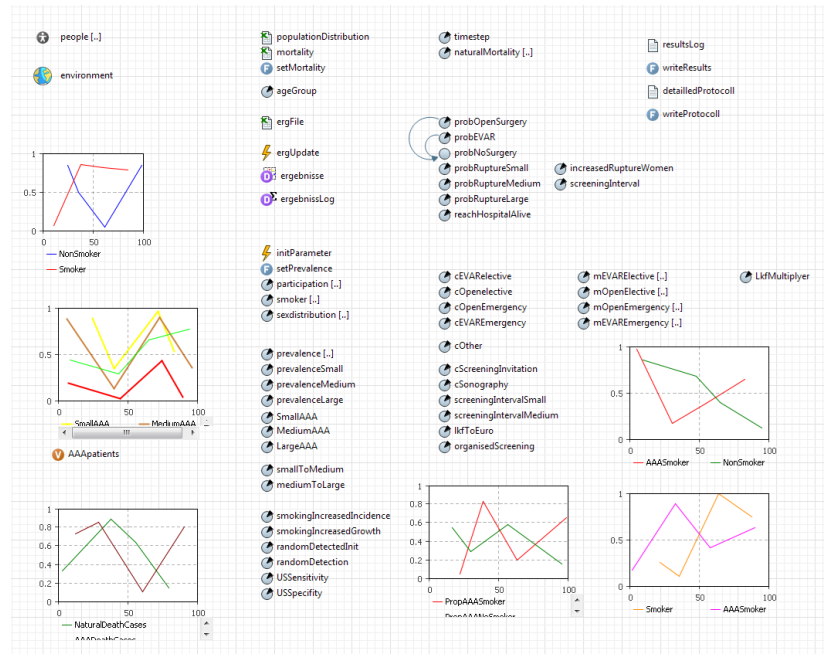

Figure 4: Implementation of the model: Main

Another problem which we do not fully understand till now is the non-linear growth of computation time when increasing the number of agents, although there is no interaction between them. Future work certainly includes investigation of such effects and code optimization.

\section{VALIDATION AND LIMITATIONS}

The model uses many different data sources, most notably reimbursement data from the Main Association of Austrian Social Security Organizations which, although very comprehensive, inherits data errors or lacks epidemiological information, especially concerning risk factors, that is needed for the accurate mathematical description of the model. This information is gained from other sources, especially published studies about AAA. Data heterogeneity and lack of information makes model validation and clear statements about its limits, which are both discusses in this section, elemental for understanding and correctly interpreting the model results. 


\section{Urach, Zauner, Endel, Wilbacher, and Breitenecker}

\subsection{Validation}

The model consists of several modules which were validated both separately as well as within the framework of the overall model. The population module was compared with absolute numbers of the main prognosis data of Statistik Austria (Statistik Austria 2012) (note that data for parameterization for the population module origins also from Statistik Austria but are different, e.g. mortality rates, than the data used for validation). Reviewing presented data, much information seems redundant, especially considering number of cases. For diameter distribution and growth results from several studies, the most important being (Mani et al. 2011), (Brady et al. 2004), (Cornuz et al. 2004) were used for parametrization. Inclusion of further risk factors (sex, smoking) required to model these aspects very detailed. Combination from results of studies, which usually regarded only single aspects, required assumptions for their interaction which are all listed above or at the description of the concerned scenario but their marginal distributions must always fit numbers of presented studies or database evaluations. Internal validation of disease progression and treatment then was performed using results from GAP-DRG which gives an overview over reported AAA-cases and when certain events like surgery or rupture occurred in Austria with model output as well as by comparison of output of model parts with related studies which were not used for parameterization. Evidence of the internal validity for simulating current state of Austria as well as results from studies like diameter growth gives confidence when evaluating scenarios with varying assumptions and testing of organized screening strategies.

\subsection{Limitations}

Although much information from international studies, databases, and expert opinions was used to create and parameterize the model there exist certain inevitable inaccuracies. First and most importantly including women to the screened population raises requirements especially for parameterization tremendously because most cases occur among men and most studies only investigate men. Also, standard deviation for evaluations from databases is much higher for women. Quality of results is therefore higher for men.

Investigations about screening follow the EUnetHTA scheme: People with a detected AAA receive annual follow-up screenings. The model simulates 65 -year old people for a time horizon of 20 years. The model uses assumptions on smoking and smoking habits as well as surgery techniques from 2012, changes of smoking habits and progress considering surgery could influence the results.

Within the IFEDH-project other risk factors besides smoking, like cardiovascular diseases or diabetes have also been investigated for integration in an AAA model but excluded for various reasons, most commonly contradiction of several studies on the subject, still there is the possibility some of these or other risk factors are identified in future studies to have an impact on AAA development.

\section{BASE SIMULATION}

In the base case scenario it is assumed that about 50 percent of the patients who have a rupture are not registered in the GAP-DRG-database (either die on the way to hospital or even before) with a corresponding 100 percent mortality. For follow-up screening the EUnetHTA scheme, if a small or medium AAA is detected 12-monthly follow-up screening is performed and patients with large AAAs usually undergo elective surgery, is used. Screening participation is assumed to be $40 \%$ and compared with the current state as well as an hypothetical $100 \%$ participation in the base analysis.

Individual properties of agents like aorta diameter at calculation start, but also for example when an AAA is randomly detected, are drawn from probability distributions which present the observed distribution. This contingency leads to deviating simulation results (Figure 5: Discrepancy of ten base simulation runs), therefore all results are means over 100 simulation runs with the same settings when not explicitly noted otherwise.

Figure 6 shows the number of ruptures which can be prevented with introduction of organized screening under the base case assumptions. The model is also used to compare overall numbers of open and endovascular 
Urach, Zauner, Endel, Wilbacher, and Breitenecker

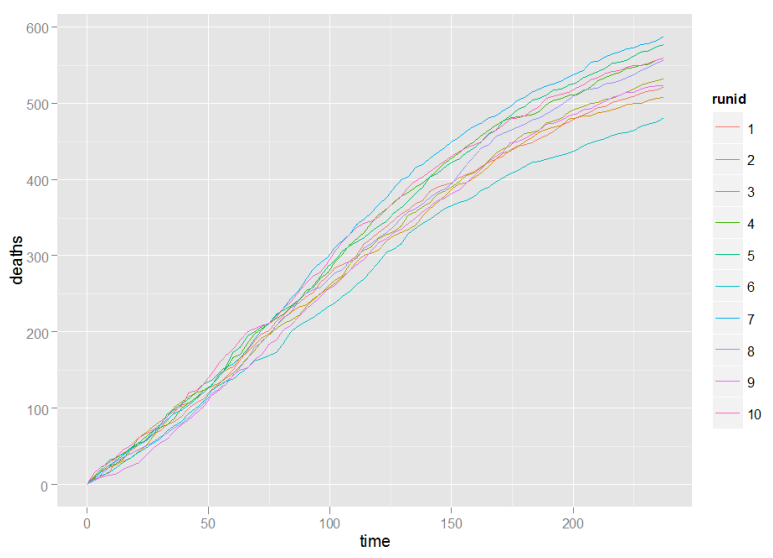

Figure 5: Deviation of simulation runs: Fatalities

surgeries both in emergency and elective cases, for different strategies as well as progressing costs and costs per life year gained which could be used as decision aid for health care decision makers.

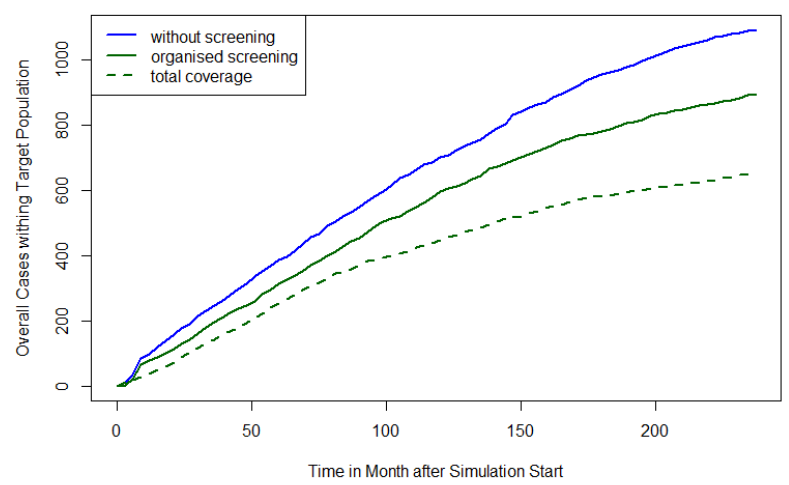

Figure 6: Comparison of ruptures of current state with 40\%, 100\% screening participation

The model is also used to compare overall numbers of open and endovascular surgeries both in emergency and elective cases, for different strategies as well as progressing costs and costs per life year gained which could be used as decision aid for health care decision makers.

\section{CONCLUSION}

The here presented model for abdominal aortic aneurysms simulates abdominal growth within the Austrian population depending on several risk factors. It is used to compare organized screening strategies but also allows, which was a primary goal, assessment of changes of other factors like smoking which have an impact on the growth of the aorta and therefore providing the possibility to explore smoking cessation programs and compare them with screening or improved treatments, e.g. lower mortality for elective surgery.

Its modular design allows integration of additional knowledge on AAA for further analysis and evaluation of screening strategies or other interventions within a short time-frame, for example if studies provide parameters for the impact of diabetes on development of AAA individuals gain additional properties and the disease progression module has to be adapted without necessity of changing the whole model structure. Furthermore the design can be adapted to evaluate other diseases with similar progression. 


\section{Urach, Zauner, Endel, Wilbacher, and Breitenecker}

Another benefit of the model is that studies of this magnitude on patients are often not possible because of temporal, logistic, ethical or other reasons. In this case the model gives insight on possible consequences of interventions and the results serve as decision aid for health care decision makers.

\section{REFERENCES}

Brady, A. R., S. G. Thompson, F. G. R. Fowkes, R. M. Greenhalgh, and J. T. Powell. 2004. "Abdominal Aortic Aneurysm Expansion : Risk Factors and Time Intervals for Surveillance". Journal of the American Heart Association 14(4):343-49.

Cornuz, J., C. S. Pinto, H. Tevaearai, and M. Egger. 2004. "Risk factors for asymptomatic abdominal aortic aneurysm". European Journal of Public Health 110:16-21.

EUnetHTA 2012. "EUnetHTA Core Information". http://meka.thl.fi/htacore/106.aspx.

Group, T. M. A. S. S. 2002. "The Multicentre Aneurysm Screening Study (MASS) into the effect of abdominal aortic aneurysm screening on mortality in men: a randomised controlled trial". The Lancet 360:1531-39.

Iribarren, C., J. Darbinian, A. S. Go, B. H. Fireman, C. D. Lee, and D. P. Grey. 2007. "Traditional and Novel Risk Factors for Clinically Diagnosed Abdominal Aortic Aneurysm: The Kaiser Multiphasic Health Checkup Cohort Study". Annals of epidemiology 17(9):669-78.

Kim, L. G., S. G. Thompson, A. H. Briggs, M. J. Buxton, and H. E. Campbell. 2007. "How cost-effective is screening for abdominal aortic aneurysms?". Journal of Medical Screening 14:46-52.

Mani, K., A. Wanhainen, J. Lundkvist, and D. Lindström. 2011. "Cost-effectiveness of intensive smoking cessation therapy among patients with small abdominal aortic aneurysms". Journal of Vascular Surgery 54(3):628-36.

Statistik Austria 2012. "Statistik Austria". http://www.statistik.at.

Sweeting, M. J., S. G. Thompson, L. C. Brown, and J. T. Powell. 2012. "Meta-analysis of individual patient data to examine factors affecting growth and rupture of small abdominal aortic aneurysms". British Journal of Surgery 99:655-65.

\section{AUTHOR BIOGRAPHIES}

CHRISTOPH URACH studied Technical Mathematics at the Vienna University of Technology and specialised on Mathematical Modelling and Simulation in the field of HTA (Health Technology Assessment). He currently works at dwh simulation services in the department of health economics where he is developing applicable model structures for evaluation of health care interventions. He is also working on a $\mathrm{PhD}$ theiss supervised by Prof. Felix Breitenecker. His email address is christoph.urach@dwh.at.

GÜNTHER ZAUNER studied Technical Mathematics with a focus on mathematical methods in computer science at Vienna University of Technology. He is currently leading the working group for modeling and simulation in health technology assessment (HTA), development of simulation strategies for infectious diseases at dwh Simulation Services. He is also working on a PhD thesis supervised by Prof. Felix Breitenecker at Vienna University of Technology. His e-mail address is guenther.zauner@dwh.at.

GOTTFRIED ENDEL works at the Main Association of the Austrian Social Insurance Institutions, in the Department for Evidence Based Health Care. He is responsible for the EBM/HTA team. His field of work includes HTA, health system research, e-health, patient safety and other questions related to the Austrian social system. He is lead partner representative in EUNetHTA, HTAi, iHEA, ISPOR, G-I-N member and board member in several Austrian associations. His e-mail address is gottfried.endel@hvb.soz-vers.at.

INGRID WILBACHER works at the Main Association of the Austrian Social Insurance Institutions, in the Department for Evidence Based Health Care. Her field of work includes HTA, health system research, socioeconomic aspects and other questions related to the Austrian social system. She is involved in the 
joint action with EUNetHTA. Her e-mail address is ingrid.wilbacher@hvb.soz-vers.at.

FELIX BREITENECKER studied 'Applied Mathematics' and acts as professor for Mathematical Modelling and Simulation at Vienna University of Technology. He covers a broad research area, from mathematical modelling to simulator development, from DES via numerical mathematics to symbolic computation, from biomedical and mechanical simulation to process simulation. He is active in various simulation societies: president and past president of EUROSIM since 1992, board member and president of the German Simulation Society ASIM, member of INFORMS, SCS, etc. He has published about 350 scientific publications, and he is author/editor of 10 books He is member of the editorial board of several journals, and conference proceedings series, and since 1995 he is editor in chief of the journal Simulation Notes Europe (formerly Simulation News Europe). His email address is felix.breitenecker@tuwien.ac.at. 